\title{
Rechtsfähigkeit, Menschenwürde und Schutz des Lebens in Brasilien: eine Einleitung
}

\author{
Von Renata Carlos Steiner*
}

\begin{abstract}
This paper intends to study how the Brazilian Law and Doctrine recognize the concept of the legal capacity, taking as paradigm especially the Brazilian Constitution of 1988, the Civil Code of 2002 and a judgment of the Brazilian Supreme Court from 2008. To this end, it analyses the importance of reading the Civil Code in parallel with the Constitution and of understanding the legal concepts in connection with reality. Special importance is given to the definition of the beginning of human life and to the moment when a human being is recognized as having legal capacity. The Brazilian Civil Code establishes that the legal capacity begins at birth, but protects the rights of the unborn, known as Nasciturus. At the same time, the Brazilian Constitution proclaims the dignity of the human being as the fundament of the Democratic State. With these two notions, it is possible to sustain that Nasciturus must also be recognized as legal subjects, since their dignity cannot be ignored. On the other hand, protecting their rights and life does not mean to treat the unborn as persons, in the juridical meaning of the term. It escalates further when it comes to the definition of legal protection in relation to genetic artificial reproduction, especially at the embryotic stage. The study brings some critical analysis on the notion of legal capacity, the dignity of the human being and the protection of life in Brazilian Law, and contains comparisons with German and Portuguese Law on this matter.
\end{abstract}

\section{A. Einleitung}

Die Person und ihre Aufgabenstellung sind Grundsatzbegriffe der Rechtswissenschaft. Man kann sagen, dass die Rechtswissenschaft nur auf Grund der Existenz der Menschen besteht. Ausser der Person ist auch das Rechtsverhältnis ein essentieller Begriff der brasilianischen Rechtslehre.

Die beiden Begriffe sind zusammenverbunden. Die Existenz der Person ist eine Voraussetzung des Begriffes ,Rechtsverhältnis‘, das zwar ein Grundlagenwissen in der traditionellen

* PhD Student at Universidade de São Paulo (USP). Master's Degree in Civil Law in the Federal University of Paraná, Brazil (Universidade Federal do Paraná, UFPR - 2009). Gast researcher in the University Augsburg, Lehrstuhl von Professor Jörg Neuner (2008). Attorney at Law in Curitiba, Paraná, Brasil. E-mail: renata@dotti.adv.br. I should thank the German expert Elisete Antoniuk for the incommensurable help in the translation and review of this text, originally written in Portuguese. 
Lehre des Privatsrechts ${ }^{1}$ ist. Juristen aus verschiedenen Ländern, wie aus Brasilien und Deutschland, aber auch beispielsweise aus Portugal, behandeln die Person nach ihrer Anerkennung als subjektives Element des Rechtsverhältnisses.

In diesem Sinne definiert Manuel António Domingues de Andrade, Autor eines der besten Werke in portugiesischer Sprache über das Thema, das Rechtsverhältnis als „eine soziale Lebensbeziehung, das vom Recht reguliert wird, durch welche ein subjektives Recht einer Person (im juristischen Sinne) zuerkannt wird, und einer anderen Person eine Pflicht oder eine Unterstellung auferlegt,,. ${ }^{2}$ Die Erkenntnis dieses Begriffes als Zuteilung von Rechten und Pflichten ist ein Grundbegriff in der Lehre des Subjekts.

Die natürliche Person ist das subjektive Element des Rechtsgeschäftes ${ }^{3}$ und wird häufig als „Rechtssubjekt“" behandelt. ${ }^{4}$ Diese Begriffe haben aber keine Bedeutung wenn sie nicht in konkreten Situationen ergriffen werden. Deshalb, im weiteren Verlauf dieser Arbeit wird darauf eingegangen, wer die Rechtssubjekte in der brasilianischen Rechtswissenschaft sind und wie man rechtsvergleichend einführende Linien über die Regulierung des Themas im deutschen Bürgerlichen Gesetzbuch (BGB) ziehen kann.

Für diesen Zweck soll als Einstieg einleitend erwähnt werden, dass das brasilianische Zivilgesetzbuch von 2002 mit der folgenden Regel anfängt: „Jede Person hat die Fähigkeit, Inhaber von Rechten und Pflichten zu sein“ (Art. 1). Nach dem Wortlaut des Gesetzes sind alle Personen Rechtssubjekte und deshalb können alle als Träger von Rechten und Pflichten an Rechtsverhältnissen teilnehmen. ${ }^{5}$

Das BGB erklärt seinerseits nicht, was unter dem Ausdruck , Rechtsfähigkeit` zu verstehen ist. Es bestimmt aber im $\S 1$, dass die Rechtsfähigkeit mit der Vollendung der Geburt anfängt. Daher ist jeder geborene Mensch rechtsfähig. Dieselbe Regelung findet man in Art. 2 des brasilianischen Zivilgesetzbuch („Die Persönlicheit der Menschen fäng mit der Lebendgeburt an; die Rechte der Ungeborenen werden ab der Empfängnis gehütet").Trotz der fehlenden Gesetzbegriffe der Rechtsfähigkeit ist ihre Wichtigkeit auch in Deutschland insbesondere in dem genannten Privatrecht unbestritten. Als allgemeines Institut des Privatsrechts hat der Rechtsverhältnisbegriff eine enge Verbindung mit dem Rechtsverkehr und dasselbe gilt für den Rechtssubjektbegriff und die Rechtsverkehrssicherheit.

1 Über die Unterscheidung zwischen Privat- und Öffentliches Recht gibt es in Brasilien eine sehr bekannte Lehre über das Ende dieser Trennung insbesondere im Hinblick auf die Umwandlung vieler Privatrechtsvorschriften in die Verfassungsurkunde. Obwohl diese Trennung in manchen Situationen auf keinen Fall nicht so deutlich angesehen werden kann, wird die Unterscheidung insbesondere akademisch noch weiter benutzt.

2 Manuel A. Domingues de Andrade, Teoria da Relação Jurídica, vol. I, Sujeitos e Objetivo, Reimpressão, Coimbra, 2003, p. 02.

3 Auch die Juristischen Personen sind Rechtssubjekte, aber sie werden nicht in dieser Arbeit behandelt.

4 Über das Thema sagt Washington de Barros Monteiro, dass das erste Element in einem Rechtsverhältnis das Subjekt oder die Person ist, ohne welche das Recht nicht existieren kann. (Washington de Barros Monteiro, Curso de Direito Civil. Vol. I: parte geral. 39. Ed. São Paulo: Saraiva, 2003, p. 61).

5 Francisco Amaral, Direito Civil: introdução, Rio de Janeiro, 2006, p. 215. 
Hauptpunkt der Arbeit ist es zu beantworten, was alle diesen Begriffe in Brasilien bedeuten. Pontes de Miranda, ein klassischer brasilianischer Jurist, unterscheidet z.B zwischen der Möglichkeit, ein Rechtssubjekt zu sein, die die Person definiert, und die wirkliche Existenz eines Rechts, die zu einem effektiver Rechtssubjekt führt. Im dieser Arbeit wird dieser Unterschied durchschaubar gemacht.

Für den hier dargelegenden Zweck wird das Thema der Rechtsfähigkeit natürlicher Person im Zusammenhang mit einer Entscheidung vom obersten brasilianischen Bundesgericht analysiert. Die Arbeit beabsichtigt, einführende Linien über die Auslegung des Themas in der Rechtslehre und das vom Gericht fällende Urteil zu analysieren. Als Einstieg wird das Verständnis des Rechtssubjektbegriffs in Brasilien dargelegt.

\section{B. Das Rechtssubjekt}

Die Studie der Rechtsfähigkeit hat unermessliche Wichtigkeit. Darüber soll anerkannt werden, dass das Subjekt als unumgänglicher Bestandteil des Rechtsverhältnisses das Zivilrecht im allgemeinen und auch das Zivilgesetzbuch im ganzen prägt: Das Subjekt schliesst Verträge, begründet eine Familie, besitzt ein Grundstück, stirbt und erbt. Wie schon oben erwähnt, ist es im Zusammenhang mit der Idee des Rechtsverhältnisses eine Schlüsselfigur der Rechtswissenschaft.

Trotz seiner Wichtigkeit gibt es in Brasilien eine bedeutsame Kritik zu der Abstraktion des Rechtssubjektbegriffes, die nicht vergessen werden soll. Im Allgemeinen kann es gesagt werden, dass die fruchtbaren Lebensverhältnisse nicht in einem Begriff, der mit der Realität nicht verbunden ist, festgelegt sein können. Dies stellt die kritische Theorie des Zivilrechtes fest und hat eine besondere Bedeutung in der brasilianischen Wirklichkeit.

In Brasilien führen die grössten sozialen Missverhältnisse und sozialen Probleme zur Schlussfolgerung, dass das Rechtsubjekt schwerlich einen Vertrag friedlich und gleichmäßig schließen wird. Normalerweise wird es nur Besitzer und kein Eingentümer sein. Obwohl es sicherlich sterben wird, erbt er aber meistens nicht. In der brasilianichen Realität ist es wenig zu sagen, dass alle fähig für Rechte und Pflichten in der Zivilordnug sind, hauptsächlich wenn es annerkant wird, dass die Proklamation dieser Fähigkeit nur diskursiv und unbegleitet von Wirkungen ist. Man spricht von einem Land, wo ungefähr 53 Millionen Leute monatlich mit weniger als die Hälfte des Mindestlohns (ungefähr 120 Euro pro Monat) überleben. ${ }^{6}$

Pontes de Miranda betont mit Recht, dass wenn man alle als Rechtsubjekte anerkennt, nimmt man an, dass eine Person immer Rechte hat. Es gibt zwar angeborene Rechte, die den unangreifbaren Kern der Person begründen. ${ }^{7}$ Trotz dieser Rechte vermutet man, dass jemand in einem Land wie Brasilien sein ganzes Leben ausserhalb der juristischen Welt stehen wird. Alle werden Personen sein, weil sie lebendig zur Welt kommen und deshalb erwarten alle ein

6 Angabe von www.brasilia.unesco.org.

7 Man spricht von Grundrechte, die allen Menschen zuerkennen sind. 
wirksames Rechtssubjekt zu sein, weil ,eine Person zu sein' nicht unbedingt bedeutet, Rechte zu haben, sondern die Möglichkeit, Inhaber von Rechten zu sein.

Die abstrakte Vorschrift des Zivilgesetzbuches, die besagt, dass alle mit der Vollendung der Geburt fähige Träger von Rechten und Pflichen sind, wiederholt fast wortwörtlich die Vorschrift des Art. 2. aus dem Zivilgesetzbuch von 1916. Unter starkem Einfluss des deutschen Rechts hat das Zivilgesetzbuch in Brasilien auch einen allgemeinen Teil, der mit dem Begriff der Personlichkeit anfängt. Um das brasilianisches Rechts zu verstehen, sind aber einige Vorbemerkungen über die Verfassungsordnung von 1988 nötig, die die Menschenwürde zum ersten Mal als Grundlage des demokratischen Rechtsstaates in Brasilien begründet hat. Da das Rechtssubjekt ein Grundbegriff im Privatrecht ist, muss er gemä $\beta$ dem Grundgesezt verstanden werden.

\section{Verfassungsmäßige Verständnis des Rechtssubjektbegriffes}

Wie oben erwähnt, findet die Lehre des Verfassungszivilrechtes umfassenden Schutz im brasilianischen Zivilrecht, hauptsächlich durch die Lehre von Luiz Edson Fachin und Gustavo Tepedino unter starker Einfluss von Pietro Perlingieri und die italienische Lehre. In diesem Zusammenhang führt die Kritik zur Abstraktion der Rechtsbegriffe zu einer zentralen Stelle, indem man erkennt, dass der Schutz des Menschen im Einverständnis mit dessen existenziellen notwendigen Bedürfnissen gesichert werden muss.

Nach der Ansicht von Gustavo Tepedino muss der Schutz der Person, der mit dem Rechtsfähigkeitsbegriff eng verbunden ist, sich einer neuen Lektüre unterziehen, die die Verfassungsgebote privilegiert. ${ }^{8}$ Aus diesem Grund sagt man, dass die Änderung der Paradigmen zu einer Neuschreibung des Begriffes führt. Unter diesem Verständnis wird die Person nicht mehr lediglich als möglicher Träger von Rechten und Pflichten, die nur mit Vermögensituationen verbunden sind, geschützt.

Zweifelsohne kann gesagt werden, dass der klassische Rechtssubjektsbegriff in enger Verbindung mit der Willensfreiheit steht. Diese Schlussfolgerung stützt sich auf der Tatsache, dass die Rechtsfähigkeit nicht nur im brasilianischen, sondern auch im deutschen Recht der Person die Teilnahme am Rechtsverkehr mit Rechten und Pflichten ermöglicht. ${ }^{9}$ Die Person kann nur am Rechtsverkehr teilnehmen, wenn und weil sie rechtsfähig ist. ${ }^{10}$

In beiden Rechtsordnungen kann auch betont werden, dass die Anerkennung der Rechtsfähigkeit verfassungsrechtliche Grundlagen hat, insbesondere wegen der Menschenwürde. Sowohl in der brasilienische Verfassung (Art. 1, Absatz III) als auch im

8 Gustavo Tepedino. Crise de fontes normativas e técnica legislativa na parte geral do Código Civil de 2002. In: TEPEDINO, Gustavo (coord.). A parte geral do Novo Código Civil: estudos na perspectiva civil-constitucional. $3^{\text {a }}$ ed. Rio de Janeiro: Renovar, 2007, p. XXIII.

9 Karl Larenz e Manfred Wolf, Allgemeiner Teil des Bürgerlichen Rechts, 9. Auflage, München 2004, p. 99.

10 Karl Larenz e Manfred Wolf, Note 8, p. 99. 
deutschen Grundgesetz (Art. 1) steht die Menschenwürde als ein Grundsatz des Demokratischen Rechtsstaats.

Über das Thema behaupten Larenz/Wolf, dass die Menschenwürde gebietet, allen Menschen unabhängig von Geschlecht, Rasse, Religion, Herkunft, Neigungen oder sonstigen Unterschieden spätestens mit der Geburt die Rechtsfähigkeit zuzuerkennen. ${ }^{11}$ Im Sinne des $\S 1$ BGB begründet die Vollendung der Geburt das Rechtssubjekt.

Um zu sagen, dass diese Fähigkeit jedem Mensch zukommt, bedeutet, dass das Recht keine besondere Auflage ausser der Geburt aufstellt, um diesem Fähigkeit zuzuerkennen. In diesem Sinne soll der Zusammenhang zwischen dem Schutz des Menschen als Rechtssubjekt und der Menschenwürde nachvollziehbar sein. Fraglich ist es aber, ob diese formelle Bestätigung die Möglichkeit bietet, die Menschenwürde zu konkretisieren. Um diese Frage zu beantworten, stellt die Verfassungszivilrechtslehre in Brasilien eine kritische neue Auslegung des Begriffs dar.

Die Menschenwürde setzt voraus, die Rechtsfähigkeit etwas weiter zu verstehen, als nur die Möglichkeit, Inhaber von Rechten und Pflichten sein zu können. Man spricht von einem ethischen Inhalt der Person, unter welchem sie nicht abstrakt gedacht werden könnte. ${ }^{12}$ Das Verfassungszivilrecht versteht, dass die Teilnahme im Rechtsverkehr nur ein Teil der Persönlichkeit ist.

Natürlich verneint das Verfassungszivilrecht nicht die Wichtigkeit der Rechtssubjekte. Die Kritik beruht nicht auf den lediglichen Begriff des Subjkets, sondern auf dessen abstrakte Auslegung, insbesondere auf eventuelle Unvereinbarkeit mit dem konkreten Leben. Über diese Entfernung betont mit Recht Luiz Edson Fachin, dass beide Begriffe - die Person und das Rechtsverhältnis - wegen ihrer Kategorisierung von den tatsächlichen Erfahrungen und Beziehungen der Menschen aussgeschlossen werden. ${ }^{13}$

Die brasilianische Verfassung von 1988 hat nach fast 25 Jahren undemokratischer Erfahrung die Grundrechtsgarantie anerkannt, unter welcher das Gleichheitsgebot zu unterstreichen ist. Nach dem Wortlaut des Art. 5 brasilianischer Verfassung sind alle Menschen ohne irgendwelche Unterscheidung vor dem Gesetz gleich. Der hier vermerkte Gleicheitssatz gebietet, tatbeständlich Gleiches rechtlich gleich zu behandeln und die Ungleichheiten zu berücksichtigen. Dies bedeutet, dass dieser Gleichheitsgrundsatz nicht als Vorwand zur Beibehaltung von soziallen Missverhältnissen angewendet werden soll.

Von dieser Anerkennung an wird das Rechtssubkjekt im Zusammenhang mit den verschiedenen Lebensverhältnissen behandelt. Der Mensch wird z.B als Verbraucher, Kind, Junge, Frau und ältere Personen behandelt. ${ }^{14}$ Bestimmte Gruppen, und deshalb bestimmte

11 Karl Larenz e Manfred Wolf, Note 8, p. 100.

12 Luiz Edson Fachin, Note 11, p. 31.

13 Luiz Edson Fachin, Note 11, p. 85.

14 Diese Gruppen haben eine besondere Stellung im brasilianischen Recht und werden gesetzlich geregelt. Zu erwähnen sind das Verbrauchergesetz (Lei 8.078/90), das Kind- und Jugendgesetz (Lei 8.069/90), die Frauengesetz (11.340/2006) und der Ältere hat ein besonderes Statut. 
Rechtssubjekte, sollen vom Gesetzgeber mit Rücksicht auf die Verfassungsgebote grundsätzlich geschützt werden.

Auch vor der brasilianischen Verfassung von 1988 wurde das Gleichheitsgebot als Grundlage des Rechtssubjektbegriffes ausgearbeitet. Beispielsweise kann man die Lehre von Clovis Bevilaqua vor Augen haben, als er im Jahre 1940 belehrte, dass das Gebot des Art. 2 Zivilgesetzbuch von 1916 ein juristisches Werk darstellte, dass allen Menschen die Möglichkeit der Teilnahme am Rechtsverkehr unabhängig von deren Geschlecht oder Herkunft gebietet. ${ }^{15}$

Das Gleichheitsgebot als Inhalt der Verfassung bedeutet nicht, dass der Begriff nur einen neuen Stellenwert hat, sondern bezeichnet seine Aufstellung als Grundlage des Rechtsstaates. Ihre Auslegung kann nur im Einklang mit der ganzen Verfassungsordnung erfolgen. Es ist die oben erwähnte Anerkenung von der substanziellen oder materiellen Gleichheit.

\section{Rechtssubjekte, Rechtsfähigkeit und Rechtspersönlichkeit: Abgrenzung der Begriffe}

Wie oben bezeichnet, hat der Rechtssubjektbegriff eine enge Verbindung mit dem Rechtsfähigkeitsbegriff. In der brasilianischen Rechtswissenschaft wird die Rechtsfähigkeit in zwei verschiedenen Sinnen ausgearbeitet: Im engeren Sinne als Delikts- oder Vertragsfähigkeit und im weiteren Sinne, der besser als Persönlichkeit verstanden werden soll. Deshalb sagt man, dass alle rechtsfähig sind, aber nicht alle können selbst am Rechtsverkehr teilnehmen. ${ }^{16}$

Man spricht von relativer und absoluter Rechtsfähigkeit. Das Wort Persönlichkeit wird häufig als Ersatz für Rechtsfähigkeit im weiteren Sinne benutzt: „Die Persönlichkeit bedeutet dasselbe wie ,Rechtsfähigkeit zu haben“ oder die Möglichkeit, Rechtssubjekt zu werden“. ${ }^{17}$ Aber es soll bemerkt werden, dass diese zwei Begriffe nicht genau dieselbe Bedeutung haben.

Die Persönlichkeit, die allen Menschen zuerkannt ist, bezeichnet die Gelegenheit der Rechtssubjekte, am Rechtsverkehr teilzunehmen. ${ }^{18}$ In einer verfassungszivilrechtlichen Auslegung ist esmöglich zu betonen, dass die Persönlichkeit einen Bestandteil hat, der weiter außer dem ledigen Begriff der Rechtsfähigkeit zu verstehen ist. Die Person soll nicht nur als

15 Clóvis Bevilaqua. Theoria Geral do Direito Civil. 5 a edição. Actualizada por Achilles Bevilaqua. Rio de Janeiro: Editora Francisco Alves, 1951, pp. 83-95.

16 Die deutsche Rechtslehre behandelt die Rechtsfähigkeit auch unabhängig von der Möglichkeit, selber die Rechte und Pflichten wahrzunehmen: Jeder Mensch ist rechtsfähig, gleichgültig ob er selber diese Rechtsfähigkeit benutzen kann. (Ulrike Pauckstadt-Maihold. Rechtsfähigkeit. In: JA 1994, Heft 10, p. 378).

17 Pontes de Miranda, Tratado de Direito Privado, Tomo I, São Paulo 1954, p. 154. Der Jurist betont, dass Rechtsfähigkeit und Persönlichkeit die gleiche Bedeutung haben.

18 Francisco Amaral, Note 5, p. 216. Man kann sagen, dass am Rechtsverkehr teilzunehmen braucht man nicht unbeding Persönlichkeit zu besitzen, sondern einfach Rechtsfähigkeit. Es gibt in Brasilien Organisationen die keine Persönlichkeit haben, aber die am Rechtsverkehr teilnehmen können, wie z.B die Erbmasse. 
möglicher Träger von Rechtsverhältnissen anerkannt werden. ${ }^{19}$ Deshalb werden die sogennante Persönlichkeitsrechte geschütz.

Für einige Autorenin Brasilien wäre die Rechtsfähigkeit ein Bestandteil der Persönlichkeit. Diese wird als die Zusammenstellung aller Gewalten von einer Person anerkannt. ${ }^{20}$ Das betont z. B. Francisco Amaral, der beide Begriffe in einer engen Verbindung versteht, die aber nicht als Synonym ausgearbeitet werden können. ${ }^{21}$ Unter diesem Verständnis wird die Rechtsfähigkeit als der Persönlichkeitsmaßstab definiert, weil jemand mehr oder weniger rechtsfähig sein kann, aber nie mehr oder weniger Person sein kann. ${ }^{22}$

Und dasselbe betont das Portugiesische Recht. Die Persönlichkeit, die jedem zuerkannt wird, bedeutet die abstrakte Möglichkeit der Teilnahme am Rechtsverkehr. Die Rechtsfähigkeit bedeutet ihrerseits, dass jemand am Rechtsverkehr teilnehmen kann, wenn dieser nicht untergesagt ist. Die wirkliche und konkrete Teilnahmemöglichkeit, und hier spricht man nicht von Handlungsfähigkeit, hängt vom Rechtsverhältnis und den Lebensbeziehungen ab. ${ }^{23}$

Sie sind in Brasilien zwei verschiedenen aber miteinander engverbundenen Begriffe. Man kann nicht von Rechtspersönlichkeit ohne Rechtsfähigkeit sprechen. Jede Person wird als Rechtspersönlichkeit anerkannt und deshalb wird ihr die Rechtsfähigkeit zuerkannt. Die Person, wie vielmals gesagt wurde, soll unter der Menschenwürde behandelt werden, ${ }^{24}$ deshalb sagt man, dass jede als Persönlichkeit anerkannt werden soll.

Es ist klar, dass die in Deutschland so gennante Rechtsfähigkeit in Brasilien besser als Persönlichkeit (Personalidade) übersetz werden soll, und dies gilt auch im portugiesischen Recht. Wie bereits gesagt, ist unter dem Begriff Rechtspersönlichkeit auch die Rechtsfähigkeit $\mathrm{zu}$ verstehen.

In diesem Zusammenfassung können drei Eigenschaften oder Grundlagen der natürlichen Person unterstrichen werden: (i) jeder Mensch ist eine Person wegen seiner Existenz und deshalb ist er fähig, Träger von Rechten und Pflichten zu sein; (ii) alle haben dieselbe Rechtspersönlichkeit und die Fähigkeit, an Rechtsverhältnissen teilzunehmen; (iii) die Persönlichkeit eines Menschen ist unverzichtbar. ${ }^{25}$

So setzt der Art. 2 des brasilianischen Zivilgesetzes voraus, dass die Persönlichkeit erst mit der Geburt anfängt,, wie ober erwähnt. Auch im BGB wird der Beginn der Rechtsfähigkeit an der Vollendung der Geburt geknüpft.

19 Silvio Rodrigues. Direito Civil. v. 1. São Paulo: Saraiva, 2003, p. 02.

20 Washington de Barros Monteiro, Note 4, p. 64.

21 Francisco Amaral, Note 5, p. 218.

22 Francisco Amaral, Note 5, p. 218. Dasselbe ist von Clovis Bevilaqua dargestellt: "Die Rechtsfähigkeit ist die Ausweitung der Handlungsfähigkeiten der Persönlichkeit”. (Note 14, p. 171).

23 Carlos Alberto da Mota Pinto, Teoria Geral do Direito Civil, 4 a ed, Coimbra 2005, p. 221.

24 Wie Carlos Alberto da Mota Pinto betont, ist die Rechtspersönlichkeit ein grundsätzliches Erfordernis der Menschenwürde (Carlos Alberto da Mota Pinto, Teoria Geral do Direito Civil, 4 a ed., Coimbra 2005, p. 198).

25 Francisco Amaral, Note 5, p. 219. 
In Brasilien soll die Regelung des Art. 1 (Zivilgesetzbuch) in Verbindung mit der vom Art. 2 ausgelegt werden. Alle sind doch fähig, Inhaber von Rechten und Pflichten zu sein; aber diese Fähigkeit fängt mit der Geburt an. Auf den ersten Blick könnte man sagen, dass nur wer lebendig geboren wird, rechtsfähig wäre.

Einschränkungen der Persönlichkeit werden nicht in der brasilianischen Rechtsordnung anerkannt. Mit der Vollendung der Geburt trifft man ein rechtsfähiges Subjekt, sonstige Unterschiede haben keinerlei Bedeutung. Nur die Fähigkeit, selber die Rechte und Pflichten wahrzunehmen, kann beschränkt werden. Man spricht von Handlungsfähigkeit.

Ausser in Brasilien wird diese Lösung auch im italienischen Codice Civile und im portuguiesichen Zivilgesetzbuch geregelt. ${ }^{26}$ Mit der Geburt erlangt jeder Mensch die Persönlichkeit- bzw. Rechtsfähigkeit. Es ist gesetzlich nicht erforderlich zu berücksichtigen, wie lange das Kind nach der Geburt gelebt hat. Auch wenn es nur kurz gelebt hat, wird die Rechtsfähigkeit ihm zuerkannt. ${ }^{27}$

Nach h.M. versteht man unter Geburt, ausser dem vollendeten Austritt des Kindes aus dem Mutterleib, auch eine einzige Atmung oder andere Zeichen des Lebens wie Selbstbewegung. Die Rechtslehre spricht normalerweise vom Eintritt der Luft in die Lungen als der Zeitpunkt, in dem das Rechtssubjekt begründet wird. ${ }^{28}$ Das bedeutet, dass vor dem Gesetz dem Kind Rechte und Pflichten verliehen sind, auch wenn es alsbald nach der Geburt stirbt. $^{29}$

Mit Rücksicht auf alle diese Gebote gibt es manche Fragen, für die man schwer eine Lösung findet. Sonderlich wichtig sind die Rechtsprobleme des noch nicht geborenen und bereits gezeugten Kindes, des so genannten Nasciturus. Fraglich ist es, ob der Nasciturus schon ein Rechtssubjekt wäre, insbesondere wegen der Anerkennung, dass er nicht schutzlos bleiben kann. Es muss auch vermerkt werden, dass die brasilianische Rechtsordnung ihm Rechte gewährt, obwohl die Persönlichkeit bwz. Rechtsfähigkeit nun erst mit der Geburt anfängt. In diesem Zusammenhang könnte man von Rechten ohne Subjekt sprechen und man fragt sich, wie der gesetzliche Ausdruck „die Rechte der Ungeborenen werden ab der Empfängnis geschützt“ zu interpretieren ist.

\section{E. Der Nasciturus}

Da die Prämisse und Schlüsselkonzepte dieser Arbeit schon ausgearbeitet wurden, wendet sich die Studie jetzt an den Schutz des Nasciturus. ${ }^{30}$ Darüber ist es anzuerkennen, dass man

26 Carlos Alberto da Mota Pinto, Note 23, p. 202.

27 Pontes de Miranda sagt, dass das Abschneiden der Nabelschnur nicht erforderlich ist, so wie die Durchführbarkeit des Kindes. (Pontes de Miranda, Note 16, p. 217).

28 So lehrt z.B. Francisco Amaral, Note 5, p. 219.

29 Karl Larenz e Manfred Wolf, Note 8, p. 103.

30 Nasciturus werden wie diejenige beschreiben, die geboren werden sollen (Rubens Limongi França. Instituições de Direito Civil. 2 a ed. São Paulo: Saraiva, 1991, p. 48). Sie sind die schon Erzeugten, aber noch nicht Geborenen. 
selbst im 21. Jahrhundert nicht sicherlich behaupten kann, wann das Leben anfängt. ${ }^{31}$ Trotz dieser Unmöglichkeit ist es zu merken, dass der Schutz des Nasciturus im Zusammenhang mit dieser Frage zu verstehen ist. ${ }^{32}$ Bemerkenswert ist es, dass die Anerkennung der Rechtsfähigkeit nicht unbedingt mit der Lebendgeburt erfolgen soll. Das unterliegt der Willkür des Gesetzgebers. Das Problem des Nasciturus kann und soll nicht nur unter rechtlichem Gesichtpunkt beanwortet werden. Verschiedene Wissenschaften wie Biologie und Medizin und andere Kenntnisse wie die Philosophie sollen in Erwägung gezogen werden. Trotz der Unmöglichkeit, eine definitive Antwort zu geben, ist die Wichtigkeit der Frage unbestreitbar.

Nicht alle Rechtsordnungen legen fest, dass die Rechtsfähigkeit erst mit der Geburt anerkannt werden soll. Das argentinische Zivilgesetzbuch z.B. verlangt im Art. 70, dass „die Persönlichkeit mit der Empfängnis beginnt, aber ihre Wirksamkeit häng von der Geburt ab“. Manche Juristen sehen als Rechtsgebungspolitik die Bestimmung von verschiedenen Zeitpunkten, ab denen die Rechtsfähigkeit anerkannt wird. ${ }^{33}$

Es gibt in der brasilianischen Rechtslehre, wie Clovis Bevilaqua betont, zwei Lehren, die den Anfang der Rechtsfähigkeit begründen.

Eine erste Lehre, die auf den ersten Blick vom brasilianischen Gesetzgeber übernommen wurde, versteht, dass die Rechtsfähigkeit nur mit der Geburt anfängt. Für diese Lehre hat der Nasciturus keine Rechte sondern Antwartschaftsrechte. ${ }^{34}$ Eine andere Doktrin stellt die Rechtsfähigkeit auf die Empfängnis ab, und in diesem Sinne erkennt dem Nasciturus Rechte zu.

In der brasilianischen Rechtsordnung werden Rechte ausdrücklich vom Gesetz des Nasciturus anerkannt (Art. 2 Zivilgesetzbuch spricht von Rechten und nicht bildenen Rechte). Das gilt insbesondere im Erbrecht, da das schon gezeugte aber noch nicht geborene Kind die Fähigkeit hat, Erbe zu sein. Viele brasilianische Gerichte bejahen die Möglichkeit, dass der Nasciturus selber Vaterschaftsklage einreichen kann. Es wird ihm auch Unterhaltungsanpruczugesprochen.

Wegen dieser Regelung, die dem Nasciturus Rechte anerkennt, betonen einige Juristen, dass sie Rechte besitzen und nicht ledige Inhaber von bildenen Rechten sind. Unter diesem Verständnis soll die Persönlichkeit auch dem Nasciturus zuerkannt werden. ${ }^{35}$

Herrschende Meinungen unterstreichen aber, dass der Nasciturus keine Persönlichkeit besitzt, denn diese fängt erst mit der Geburt an. In diesem Zusammenhang lehrt aber Clovis Bevilaqua, dass es logischer wäre, wenn man die Persönlichkeit des Nasciturus anerkennen

31 Über diese Unmöglichkeit siehe Gustavo Tepedino, Heloisa Helena Barbosa e Maria Celina Bodin de Moraes. Código Civil Interpretado de acordo com a Constituição da República, Rio de Janeiro: Renovar, 2009, p. 07.

32 So betont Silvio Rodrigues, Note 18, p. 03.

33 Francisco Amaral, Note 5, p. 222.

34 Clovis Bevilaqua, Note 14, p. 178.

35 Da Rechte zu haben von der Anerkennung der Persönlichkeit abhängt. (Francisco Amaral, Note 5, p. 221). 
würde. ${ }^{36}$ Hauptargument ist es, dass die Anerkennung eines Rechts oder Pflicht an der Existenz der Rechtsfähigkeit geknüpft wird.

Nach Carlos Alberto da Mota Pinto aus Portugal gibt es in diesem Land auch zwei Lehren diesbezüglich. Nach der Meinung einiger Lehrer wäre die Existenz eines Rechts ohne das entsprechende Subjekt nicht vorstellbar. Aus diesem Grund wird der Rechtsschutz des Nasciturus von der späteren Lebendgeburt abhängig gemacht. ${ }^{37}$ Nach anderer Meinung wird anerkannt, dass man wegen der vorläufigen Situation des nocht nicht geborenen, aber bereits gezeugten Kindes, von Rechten ohne Subjekt sprechen könnte.

Unaghängig der Lehre soll man merken, dass das brasilianische Zivilgesetzbuch Rechte an den Nasciturus voraussetzt und, im weiteren Sinne, ihn nicht schutzlos bleiben lässt. Auch die Juristen, die die Rechtsfähigkeit oder Persönlichkeit des Nasciturus verneinen, betonen, dass sie geschützt werden soll. Eine wichtige Frage ist es, ob sie geschützte Subjekte oder einfach ein Gegenstand sind, der vom Recht geschützt wird.

Da dem Nasciturus Rechte zuerkannt werden, befürworten manche Juristen in Brasilien, dass sie schon vor der Geburt rechtsfähig sind. So betont Rubens Limongi: „Rechte zuzuerkennen bedeutet auch Rechtsfähigkeit zunzuerkennen. Da Rechtsfähigkeit anerkannt wird, soll auch die Persönlichkeit nicht verneint werden“. ${ }^{38}$ Das ist auch die Meinung vom Francisco Amaral. Für ihn hat der Nasciturus eine so genannte formelle Persönlichkeit.

In dieser Diskussion kann man nicht einfach bestätigen, dass das brasilianische Gesetzbuch im Art. 2 nur das schon geborene Kind schützt. ${ }^{39} \mathrm{Im}$ Verfassungszivilrecht wird mit Recht betont, dass die Auslegung dieses Artikels nur unter den Grundrechten erfolgen soll. Dies gilt insbesondere für den verfassungsrechtlichen Schutz vom Leben (Art. 5 caput Brasilianische Verfassung).

Die Lehre vom Verfassungszivilrecht betont, dass, wegen der Notwendigkeit, das Leben des Nasciturus zu schützen, muss der Rechtssubjektbegriff zum neuen Verständnis führen. Für einige Juristen in Brasilien bedeutet die verfassungsrechtliche Anerkennung des Schutzes vom Leben auch, dass alle Menschen und auch die noch nicht Geborenen Inhaber von Rechten sind und deshalb als Persönlichkeit anerkennen werden sollen.

Schließlich soll betont werden, dass trotz der Regel des Art. 2 Zivilgesetz die Rechtspersönlichkeit dem Nasciturus in Brasilien zuerkannt wird. Klar ist es keine einstimmige Lehre, aber eine der wichtigsten.

Außer dem Schutz des Nasciturus hat auch große Bedeutung der Schutz des Embryos, insbesondere des durch künstliche Befruchtung im Rahmen der modernen Reproduktionsmedizin Erzeugten. Und in Bezug auf diese Embryonen ist der Streit noch komplizierter.

36 Clovis Bevilaqua, Note 14, p. 178. Es soll aber gesagt werden, dass die Juristen, die die Persönlichkeit ab der Befruchtung anerkennen, betonen, dass die Rechtsfähigkeit sich erst mit der Geburt begründet. (Rubens Limongi França. Note 29, p. 47-48).

37 Carlos Alberto da Mota Pinto, Teoria Geral do Direito Civil, 4 a ed, Coimbra 2005, p. 197.

38 Rubens Limongi França, Note 29, p. 50.

39 Luiz Edson Fachin, Note 11, p. 33. 
Normalerweise werden die Embryonen in Brasilien nicht als Nasciturus anerkannt. In Brasilien ist es umstritten, weil in Portugal z.B. die Embryonen als Nasciturus anerkannt werden. ${ }^{40}$

Nasciturus werden in Brasilien als ,diejenige, die zur Welt kommen sollen` definiert und deshalb schon im Mutterleib sind. Embryonen sind seinerseits diejenige, die noch nicht in den Mutterleib implantiert worden sind. Es gibt auch Juristen, die betonen, dass die Embryonen schon Nasciturus sind, obwohl sie von der Leibesfrucht zu unterscheiden sind. ${ }^{41}$

In Brasilien gibt es kein Gesetz zum Schutz von Embryonen, obwohl es besondere Regelungen zu deren Schutz gibt (Lei 11.105/2005). Im Art. 5 des brasilianischen Biotechnologieschutzgeseztes wurden Anforderungen auf die Instrumentalisierung dieser Embryonen für Forschungszwecke ausdrücklich gestellt. Im Allgemeinen gibt es zwei Möglichkeiten: (i) wenn sie schon drei Jahre lang tiefgekühlt sind; oder (ii) wenn sie nicht mehr zur künstlichen Befruchung in eine Frau übertragen werden können.

Eine Verfassungswidrigkeitsklage gegen diese Regelung wurde vom brasilianischen Generalstaatsanwalt vor dem obersten Bundesgericht eingereicht. Der vom Anwalt ausdrückliche vorgestellte Hauptgrund geht davon aus, ab wann das menschliche Leben vom Recht anerkannt wird. Deshalb argumentiert er, dass der Art. 5 des Gesetzes wegen der Verletzung der Grundrechte, insbesondere der Menschewürde und des Schutzes vom Leben verfassungswidrig sei. Er plädiert ausdrücklich, dass das Leben bereits ab der Befruchtung beginnt. Diese Tatsache ist auf die Kenntnisse im Bereich der Biologie und Medizin zurückzuführen. Er betonte auch, dass diese Regelung sicherlich verfassungswidrig sei, da sie den Schutz vom Leben verletzt. Ein Embryo soll als menschliches Leben anerkannt werden, und deshalb bedeutet seine Verletzung auch die Verletzung des Rechtstaates und der Grundrechte, die von ihm erkennntbar sind. Seiner Meinung nach verstößt die Benutzung des Embryos in der Forschungsarbeit gegen das Recht zum Leben, das auch dem Embryo zuzuerkennen ist.

Larenz lehrt, dass es verschiedene Fragen in Bezug auf den Beginn des menschlichen Lebens gibt, so wie ab wann es vom Recht geschützt wird und ab welchem Augenblick an der Mensch rechtsfähig ist.. ${ }^{42}$ Sie sind doch unterschiedliche Fragen. Der Schutz des menschlichen Lebens hängt nicht von der Anerkennung der Rechtsfähigkeit oder Rechtspersönlichkeit ab.

Trotz diesem Unterschied kann auch gesagt werden, dass beide Fragen im Zusammenhang stehen. ${ }^{43}$ Dieser Zusammenhang wurde vom obersten brasilianischen Bundesgericht entschieden.

40 Antonio Menezes Cordeiro, Tratado de Direito Civil Português. Parte Geral, tomo I. Coimbra: Almedina, 2000, p. 290.

41 Silmara Juny de Abreu Chinelatto, Estatuto Jurídico do Nascituro: a evolução do Direito Brasileiro. In: Diogo Leite de Campos e Silmara Juny de Abreu Chinelatto. Pessoa Humana e Direito. Coimbra: 2009 , p. 419.

42 Karl Larenz e Manfred Wolf, Note 8, p. 107.

43 In Portugal wird betont, dass die Rechtspersönlichkeit und das menschliches Leben im Zusammenhang sind und deshalb können sie nicht getrennt werden. 


\section{F. Die Geburt der Person: Das Verständnis vom brasilianischen obersten Bundesgericht ${ }^{44}$}

Was oben als Rechtsgebungspolitik bezeichnet war, ist vom brasilianischen obersten Bundesgerichts angeführt worden und deshalb sollte das Gericht 2008 den Streit darüber entscheiden, wann der Schutz vom Leben anerkannt wird. Als das Gericht über die Verfassungswidrigkeit des Art. 5 des brasilianischen Biotechnologieschutzgesezt beurteilen sollte, musste er unbedingt die Kontroverse beantworten: „Wann wird der Schutz vom Leben anerkannt?"

Um diese Frage zu antworten, haben die Minister/Richter mittelbar auch über die Anerkennung der Rechtspersönlichkeit beurteilt, da ihrer Meinung nach beide Fragen im Zusammenhang sind.

Die einfache Mehrheit der Richter vom Bundesgericht hat unter verschiedenen Gründen entschieden, dass die Embryonen zum Forschungszweck benutzt werden können. Es werden hier zwei unterschiedliche Gründe unterstrichen, um festzulegen, inwieweit das Thema noch kontrovers ist.

Vom Berichterstatter Minister Carlos Brito wurden die folgenden Punkte ausgearbeitet:

(i) Nur die natürlichen Personen, die lebendig zur Welt kommen, sind unter dem Rechtsfähigkeitsbegriff zu verstehen;

(ii) Der Art. 2 des brasilianischen Zivilgesetzes legt fest, dass die Rechtspersönlichkeit der Person erst mit der Lebendgeburt anerkannt wird;

(iii)Deshalb sollen im ersten Blick die Grundrechte, insbesondere der Schutz vom Leben, nur einem Mensch als Persönlichkeit zuerkannt werden;

(iv) Als die brasilianische Verfassung den Schutz vom Leben reguliert hat, wurden nicht nur die bereits Geborenen aber auch der Nasciturus geschützt;

(v) Der Nasciturus ist aber keine Person, sondern eine bildene Person;

(vi)Die Benutzung eines Embryos, der nicht auf eine Frau zur künstlichen Befruchtung übertragen werden kann, verletzt kein Grundrecht, da dieser Embryo kein mögliches Subjekt ist.

Vom Minister Eros Grau wurden seinerseits die folgenden Punkte abgegrenzt:

(i) Der Nasciturus ist nicht nur vom Recht geschützt, sondern ist erauch ab der Empfägnis Inhaber vom Rechten;

(ii) Da ihm Rechte zuerkannt werden, ist der Nasciturus eine Person und wird die Persönlichkeit ihm anerkannt;

(iii)Die Embryonen, die nicht in eine Frau übertragen wurden, sind keine Nasciturus und können nicht als Person anerkannt werden;

(iv) Deshalb verletzt die Benutzung des Embryos für Forschungszweck keine Grundrechte und auch nicht den Schutz vom Leben, da sie keine Person sind; 
(v) Aber dies gilt soweit die Embryonen nicht durch die Forschung vernichtet werden oder wenn sie nicht mehr in eine Frau übertragen werden können.

Im Allgemeinen war die Entscheidung des Bundesgerichts in die Richtung, dass die in vitro erzeugten Embyronen nicht als Nasciturus behandelt werden können und deshalb ist es doch möglich, sie für Forschungszweck zu benutzen.

Es wird hier nicht von der Abrechnung oder Irrtum der Urteile gesprochen und auch nicht von der Möglichkeit, Forschungsarbeit mit Embryonen durchzuführen. Die Situation des Themas ider brasilianischen Rechtswissenschaft ist noch kompliziert und man könnte sich lang darüber auseinandersetzten.

Es ist klar, dass der Schutz vom Leben auch den noch nicht Geborenen anerkannt wird, gleichgültig ob sie schon rechtsfähig sind oder nicht. Das beteutet nicht, dass keine Forschung mit den Embryonen gemacht werden kann, weil nach der Meinung des obersten brasilianischen Gerichts die Embryonen keine Nasciturus und deshalb auch keine Person sind.

Da diese Arbeit von der Rechtsfähigkeit natürlicher Personen handelt, müssen die verschiedenen Gründe und Meinungen der Minister des obersten Gerichts unterstrichen werden. Alle fanden den Konsens, dass die Embryonen keine Rechtssubjekte sind, aber dasselbe kann man nicht über die Anerkennung des Nasciturus als Persönlichkeit behaupten.

Obwohl die Verfassungswidrigkeit des Gesetzes vom brasilianischen obersten Bundesgericht beurteilt worden ist, besteht die Frage und die abgängigen Zweifel, wann der Mensch als Persönlichkeit vom Recht anerkannt wird. Nicht alle Richter haben ausdrücklich betont, dass die Rechtspersönlichkeit vor der Geburt anerkannt werden soll.

Auch zu achten ist die Regelung der Amerikanische Konvention über Menschenrechte, die sogenannte San Jose da Costa Rica Pakt. Im Art. 3 wird es ausdrücklich bestimmt, dass die Rechtspersönlichkeit allen Menschen zuerkannt werden soll. Im Art. 4 wird auch ausdrücklich geregelt, dass der Schutz vom Leben jedem Menschen ab der Befruchtung zuzuerkennen ist.

Und was unter Befruchtung in der brasilianischen Realität zu verstehen ist, bedeutet nur die in eine Frau bereits übertragenen Embryonen (sei es künstlich oder auf natürliche Weise).

\section{G. Schlussfolgerung}

Zum Schluss kann man über das Thema folgendes zusammenfassen:

I. Die Rechtsfähigkeit ist auch im brasilianischen Recht ein Grundbegriff;

II. Alle sind fähig, Inhaber von Rechten und Pflichten sein zu können und dies wird in Brasilien als Rechtspersönlichkeit anerkannt;

III. Die Anerkennung der Rechtspersönlichkeit bzw. Rechtsfähigkeit im brasilianischen Recht ist ab der Lebendgeburt festgesetzt;

IV. Von der Verfassungszivilrechtslehre soll der Begriff zu einer neuen Auslegung führen, da die Grundrechte auch dem noch nicht Geborenen zuerkannt sind; V. Gleichgültig welche Lehre angenommen wird, sind auch die Nasciturus geschützt und werden ihnen Rechte zuerkannt; 
VI. Nach der Meinung des obersten Bundesgerichts sind die Embryonen kein Nasciturus und haben auch keine Rechtspersönlichkeit, deshalb können sie dem Forschungszweck dienen, soweit sie nicht in einer Frau übertragen werden können oder wurden;

VII. Die Auslegung vom Gericht hat unermessliche Wichtigkeit für andere weiterführende Themen und aufgrund dessen kann man sagen, dass das Thema in Brasilien noch sehr unsicher und zweifelhaft ist. 